\title{
Budgetplanung
}

\section{IGeL bewerben kann sich auszahlen}

\author{
Ein Marketing-Budget kann helfen, den Kostenrahmen für Investiti- \\ onen abzustecken. Die Höhe der Ausgaben orientiert sich dabei auch \\ an individuellen Gesundheitsleistungen: Mit gezielter Werbung lässt \\ sich die Nachfrage nach ihnen steigern.
}

$\mathrm{D}$ ie Planung eines Marketing-Budgets ist für niedergelassene Ärzte bisher noch keineswegs Standard. Nach der Studie „Ärzte im Zukunftsmarkt Gesundheit 2009“ der Stiftung Gesundheit hatte etwa jede sechste Praxis im vergangenen Jahr ein Budget für MarketingAusgaben. Über die Höhe des Budgets waren die Umfrageteilnehmer ein Jahr zuvor befragt worden. Demnach lag das Budget bei Ärzten im Schnitt bei 5.500 Euro pro Jahr, was durchschnittlich etwa $1,5 \%$ des Umsatzes entsprach.

\section{Alles eine Frage der Abgrenzung}

Doch wozu braucht eine Praxis eigentlich ein Marketing-Budget? Die Studie der Stiftung Gesundheit zeigt zum Beispiel, dass die Umfrageteilnehmer zu den drei wichtigsten Marketing-Maßnahmen die Internet-Präsenz, das Personal der Praxis und das äußere Erscheinungsbild der Praxis zählen. Um auf diesen Feldern zu punkten, könnten etwa Investitionen in einheitliche Praxiskleidung, in eine professionell gestaltete Praxishomepage oder auch in Visitenkarten oder Praxisflyer sinnvoll geplant werden. Hinzu kommen natürlich klassische Werbemaßnahmen wie Veranstaltungen, Anzeigen in Printoder Online-Medien, Patientenbefragungen oder auch Recall-Maßnahmen zum Beispiel für Präventionsleistungen. Wer plant, gibt nicht zu viel Geld für Dinge aus, die eigentlich keine hohe Priorität haben. Auch eine gesetzte Obergrenze kann helfen, sich selbst bei den Ausgaben zu disziplinieren.

Zum anderen brauchen Praxischefs solche Planzahlen für eine vollständige
Finanzplanung, die für Gespräche mit Banken vor einer Kreditvergabe hilfreich sein können. Wer eine Strategie hat und weiß, wie viel Geld er wofür ausgeben will und vor allem mit welchem Ziel, der hat immer die besseren Karten.

Die Höhe der Marketing-Ausgaben orientiert sich dabei auch an den individuellen Gesundheitsleistungen (IGeL), die beworben werden sollen. Ist es eine innovative Leistung, die für viele Patienten in frage kommt? Ist es eine hochpreisige Leistung wie der Manager-Check-up? Oder ist es eher eine exotische Leistung, die vielleicht besser ausschließlich im direkten Gespräch angeboten werden sollte?

\section{Grundregeln beachten}

Welches Potenzial eine Praxis für welche IGeL-Angebote hat, lässt sich außer über Patientenbefragungen auch mit relativ geringem Aufwand in der Praxissoftware eruieren. So hilft ein Blick in die Daten der Stammpatienten (Alter, Geschlecht, Diagnosen), um herauszufinden, welche neuen IGeL-Angebote in der Praxis angeboten werden können, beziehungsweise $\mathrm{zu}$ entscheiden, welche bestehenden Selbstzahlerangebote ausgebaut werden sollen.

Je nach Patientengruppe, für die ein neues Angebot infrage kommt, kann es sich lohnen, mehr Geld für das OnlineMarketing einzuplanen als für Anzeigen in Zeitungen - um zum Beispiel auf der Praxis-Homepage bestimmte IGeL für internet-affine Jugendliche und zahlungskräftige Manager professionell zu promoten - oder auch in Bannerschaltungen auf Spezialportalen. 